\title{
Efficacy of Tolvaptan in Elderly Patients with Heart Failure and Preserved Ejection Fraction
}

\author{
Yoriyasu Suzuki, Akira Murata, Satoshi Tsujimoto, Yusuke Ochiumi, Tatsuya Ito
}

Division of Cardiovascular Medicine, Nagoya Heart Center, Nagoya, Aichi, Japan

\section{ABSTRACT}

Background: There is no known therapy with proven efficacy for improving clinical outcomes in elderly patients with heart failure (HF) and preserved ejection fraction (HFpEF). In this study, we aimed to evaluate the efficacy of tolvaptan (TLV) in elderly HFpEF patients. Methods: This retrospective observational study involved 100 consecutive elderly HFpEF patients hospitalized at the Nagoya Heart Center, Japan. Inclusion criteria were: (1) patients aged $\geq 75$ years; (2) first hospitalization secondary to HF; (3) received medical therapy for HF, without invasive treatment; and (4) clinical follow-up for $>6$ months after discharge. The primary endpoint was rehospitalization due to worsening HF, and the secondary endpoint was worsening renal function (WRF) during hospitalization and at 6 months after discharge. Sixty background-matched HFpEF patients were divided into 2 groups: with TLV therapy (TLV (+), $\mathrm{n}=29$ ) and without TLV therapy (TLV $(-), \mathrm{n}=31$ ). In the TLV (+) group, TLV therapy was continued after discharge. Clinical outcomes of these patients were evaluated. Results: Bed rest period and length of hospital stay were significantly shorter in the TLV (+) group than in the TLV (-) group. The dose of loop diuretics, mean serum creatinine levels, and incidence of WRF development were significantly lower in the TLV (+) group. Incidence of rehospitalization was also significantly lower in the TLV $(+)$ group (log-rank test; $\mathrm{p}=0.018$ ). The multivariate logistic regression analysis demonstrated that TLV therapy reduces the incidence of rehospitalization in elderly patients with HFpEF. Conclusions: TLV therapy reduced the bed rest period, length of hospital stay, and rate of rehospitalization without WRF in elderly HFpEF patients, suggesting that TLV could represent an effective therapy for this group of patients.

Keywords: heart failure, rehospitalization, renal function

\section{ARTICLE HISTORY}

Received: April 8, 2019

Accepted: May 19, 2019

\section{Yoriyasu Suzuki}

Division of Cardiovascular Medicine, Nagoya Heart Center 1-1-14 Sunadabashi, Higashi-Ku, Nagoya, Aichi, Japan 461-0015 Tel/Fax: +81527190810

E-mail: yoriyasu55@gmail.com

\section{INTRODUCTION}

In recent years, it has been estimated that $30-50 \%$ of patients with heart failure (HF) have preserved ejection fraction (HFpEF). These patients have a poor prognosis, have more frequently atrial fibrillation (AF), and are more likely to be older, women, hypertensive, and anemic compared to HF patients with reduced ejection fraction (HFrEF). ${ }^{1,2}$ The current therapy available for this group of patients includes a decrease in loading conditions, treatment of comorbidities, which can lead to a reduction in symptom severity and increase in functional capacity, thus preventing rehospitalizations. Although tolvaptan (TLV) has been previously investigated in patients with HF, there are currently few studies that have 
discussed its long-term efficacy in elderly patients with HFpEF. ${ }^{3-10}$

The aim of this study was to evaluate the short- and long-term clinical efficacy of TLV for treating elderly patients with HFpEF.

\section{METHODS}

This retrospective observational study involved all consecutive HFpEF patients hospitalized at the Nagoya Heart Center, Japan, from 2013 to 2017. Inclusion criteria for this study were as follows: (1) patients aged $\geq 75$ years; (2) first hospitalization secondary to acute decompensated heart failure (ADHF); (3) patients who had received medical therapy for HF, without invasive treatments such as cardiac surgery or catheter-based intervention; and (4) patients who were clinically followed up for $>6$ months after discharge. Exclusion criteria for this study were mechanical ventilation, cardiogenic shock, dialysis, and malignancy.

To diagnose HF, we used the Framingham criteria. ${ }^{11}$ With regard to the definition of HFpEF, we employed the European Society of Cardiology (ESC) guidelines and used Doppler echocardiography to establish the diagnoses. Echocardiography was performed within 24 hours after hospitalization. ${ }^{12}$ In this study, we defined HFpEF according to the following criteria: (1) signs or symptoms of HF; and (2) LVEF $\geq 50 \%$ as determined by trans-thoracic echocardiography.

The primary endpoint of this study was rehospitalization due to worsening $\mathrm{HF}$, and the secondary endpoints were worsening renal function (WRF) during hospitalization and at 6 months after discharge. WRF was defined as $\geq 0.3 \mathrm{mg} / \mathrm{dL}$ increase in serum creatinine level above the baseline at any time during hospitalization and at 6 months after discharge. The bed rest period was defined as the time interval between the day of admission and the day on which cardiac rehabilitation was initiated in the rehabilitation room. The congestion scale included a standard 4-point scoring system 4 in which clinical features were comprised of heart failure signs and symptoms (shortness of breath, distended jugular venous system, pulmonary congestion, and lower limb edema), and the score was evaluated at baseline and at 3 and 6 months after discharge. The congestion score was calculated by summing the individual scores.

After applying the inclusion and exclusion criteria, the patients were divided into 2 groups: with TLV therapy (TLV (+) group) and without TLV therapy (TLV (-) group). Informed consent was obtained from each patient, and the hospital ethics committee approved this study. The timing and dosage of TLV therapy of each patient were at the discretion of their attending physician.

The present study complies with the Declaration of Helsinki, and the institutional review board of our institute has approved the research protocol. Informed consent was obtained from all participants before enrollment into the study.

\section{STATISTICAL ANALYSIS}

Continuous data are presented as mean \pm SD. All differences were compared using Student's t-test and nonparametric methods. Discrete variables are expressed as counts and percentages. These were assessed using Fisher's exact test or the chi-squared test, depending on the sample size. Multivariate logistic regression analysis was applied to identify the risk for rehospitalization. Time-toevent data were summarized as Kaplan-Meier estimates according to the rehospitalization status and were compared using the log-rank test. Propensity score matching analysis was adopted for eligible patients to select the background-matched control population. In all analyses, $\mathrm{p}<0.05$ was considered to indicate statistical significance. Statistical analyses were performed using SPSS version 23 (IBM Corp., Armonk, NY, USA).

\section{RESULTS}

A total of 377 consecutive first-hospitalized ADHF patients were treated at the Nagoya Heart Center between 2013 and 2017. Among these patients, 118 had undergone invasive therapies, whereas the remaining 259 patients received medical therapy. Among these 259 patients, 100 patients were diagnosed with HFpEF. After propensity score matching analysis, we enrolled $60 \mathrm{HFpEF}$ patients into this study. The TLV (+) and (-) groups comprised 29 and 31 patients, respectively. The proportion of patients who started TLV therapy from the first day of hospitalization was $82.8 \%$, and all patients treated with TLV continued the therapy even after discharge.

The baseline characteristics and the clinical outcomes are shown in Table 1. There was no difference in the baseline characteristics between the two groups. The duration of continuous intravenous infusion using diuretics, Carperitide, and inotropes was significantly shorter in the TLV (+) group than in the TLV (-) group: TLV (+) vs. TLV $(-) ; 1.8 \pm 1.3$ days vs. $4.0 \pm 4.1$ days, $\mathrm{p}<0.0001$. The bed rest period and the length of hospital stay were significantly shorter in the TLV (+) group than in the TLV (-) group: 
TABLE 1. Baseline characteristics and clinical outcomes

\begin{tabular}{|c|c|c|c|}
\hline & $\begin{array}{c}\operatorname{TLV}(+) \\
\mathrm{n}=\mathbf{2 9}\end{array}$ & $\begin{array}{c}\operatorname{TLV}(-) \\
\mathbf{n}=\mathbf{3 1}\end{array}$ & $\mathrm{p}$ value \\
\hline Male, \% & 55.2 & 55.5 & 0.102 \\
\hline Age & $84.3 \pm 5.4$ & $85.5 \pm 5.4$ & 0.565 \\
\hline BMI & $22.6 \pm 4.9$ & $20.2 \pm 5.0$ & 0.218 \\
\hline $\mathrm{AF}, \%$ & 34.1 & 36.0 & 0.644 \\
\hline Etiology, \% & & & 0.740 \\
\hline IHD & 13.8 & 12.9 & \\
\hline VHD & 44.8 & 42.3 & \\
\hline HHD & 27.6 & 25.8 & \\
\hline Duration of continuous IV infusion, day & $1.8 \pm 1.3$ & $4.0 \pm 4.1$ & $<0.001$ \\
\hline Carperitide, \% & 27.6 & 25.8 & 0.553 \\
\hline $\mathrm{DOA} / \mathrm{DOB}, \%$ & 13.8 & 12.6 & 0.279 \\
\hline ACE-I, ARB, \% & 86.2 & 90.0 & 0.433 \\
\hline$\beta$-blocker, \% & 75.9 & 76.0 & 0.597 \\
\hline $\mathrm{CCB}, \%$ & 38.3 & 38.0 & 0.255 \\
\hline MRA, \% & 85.6 & 89.3 & 0.441 \\
\hline Bed rest period, day & $2.6 \pm 1.5$ & $4.8 \pm 3.4$ & 0.001 \\
\hline Loop dose at discharge, $\mathrm{mg}$ & $24.3 \pm 14.1$ & $41.9 \pm 14.5$ & 0.024 \\
\hline TLV dose, mg & $8.3 \pm 3.7$ & & \\
\hline $\mathrm{Cr}$ at admission, mg/dL & $1.19 \pm 0.48$ & $1.15 \pm 0.44$ & 0.288 \\
\hline NT-proBNP at admission, $\mathrm{pg} / \mathrm{mL}$ & $4015 \pm 3488$ & $4130 \pm 3061$ & 0.355 \\
\hline $\mathrm{Cr}$ at discharge, $\mathrm{mg} / \mathrm{dL}$ & $1.16 \pm 0.45$ & $1.26 \pm 0.34$ & 0.364 \\
\hline Congestion score at discharge & $0.52 \pm 1.22$ & $0.32 \pm 0.79$ & 0.736 \\
\hline $\mathrm{Hb}$ at discharge, $\mathrm{mg} / \mathrm{dL}$ & $11.5 \pm 1.9$ & $11.8 \pm 1.8$ & 0.963 \\
\hline Htc at discharge, $\%$ & $35.0 \pm 5.3$ & $36.0 \pm 5.1$ & 0.938 \\
\hline Length of hospital stay & $12.9 \pm 5.8$ & $17.5 \pm 12.5$ & 0.01 \\
\hline Loop dose at 6 months, mg & $32.5 \pm 14.2$ & $46.1 \pm 14.6$ & 0.044 \\
\hline NT-proBNP at 6 months, $\mathrm{pg} / \mathrm{mL}$ & $1305 \pm 2238$ & $2523 \pm 3061$ & 0.052 \\
\hline Cr at 6 months, mg/dL & $1.13 \pm 0.63$ & $1.43 \pm 0.55$ & 0.016 \\
\hline Congestion score at 6 months & $0.26 \pm 0.66$ & $0.83 \pm 1.23$ & 0.016 \\
\hline
\end{tabular}

MI - myocardial infarction; EF - ejection fraction; RD - renal dysfunction; MVD - multivessel disease

TLV (+) vs. TLV (-); $2.6 \pm 1.5$ days vs. $4.8 \pm 3.4$ days, p $=0.001$ for the bed rest period; $12.9 \pm 5.8$ days vs. $17.5 \pm$ 12.5 days, $\mathrm{p}=0.01$ for the length of hospital stay. The dose of loop diuretics at both discharge and at 6 months after discharge, as well as serum creatinine level at 6 months after discharge, were significantly lower in the TLV (+) group. Although there was no difference in the congestion score at discharge, it was significantly higher in the TLV (-) group at 6 months after discharge. The level of NTproBNP was also significantly higher in the TLV (-) group at 6 months after discharge. As shown in Figure 1, the incidence of in-hospital and mid-term WRF development was significantly lower in the TLV $(+)$ group: (TLV $(+)$ vs. TLV (-); in-hospital WRF, $3.4 \%$ vs. $22.6 \%, \mathrm{p}=0.013$; mid-term WRF, $11.3 \%$ vs. $25.0 \%, p=0.034$. The incidence of all-cause death and rehospitalization was significantly lower in the TLV (+) group than in the TLV (-) group: TLV (+) vs. TLV (-); all-cause death, $3.4 \%$ vs. $29.0 \%$, p = 0.013; rehospitalization, $20.7 \%$ vs. $58.1 \%, p=0.03$.

Kaplan-Meier analysis revealed that the incidence of rehospitalization was significantly lower in the TLV (+) group than in the TLV (-) group (log-rank test; $\mathrm{p}=0.018$ ) (Figure 2). As shown in Table 2, multivariate logistic regression analysis demonstrated that female sex and the administration of catecholamine during hospitalization were negative predictors of rehospitalization, and that TLV therapy significantly prevented rehospitalization (female sex: odds ratio [OR] 2.861, 95\% confidence interval [CI] 2.032-8.382, $\mathrm{p}=0.007$; administration of catecholamine during hospitalization: OR 2.559, 95\% CI 1.176-6.723, p $=0.049$; TLV therapy: OR 0.191, 95\% CI 0.116-0.717, $\mathrm{p}=$ $0.023)$. 


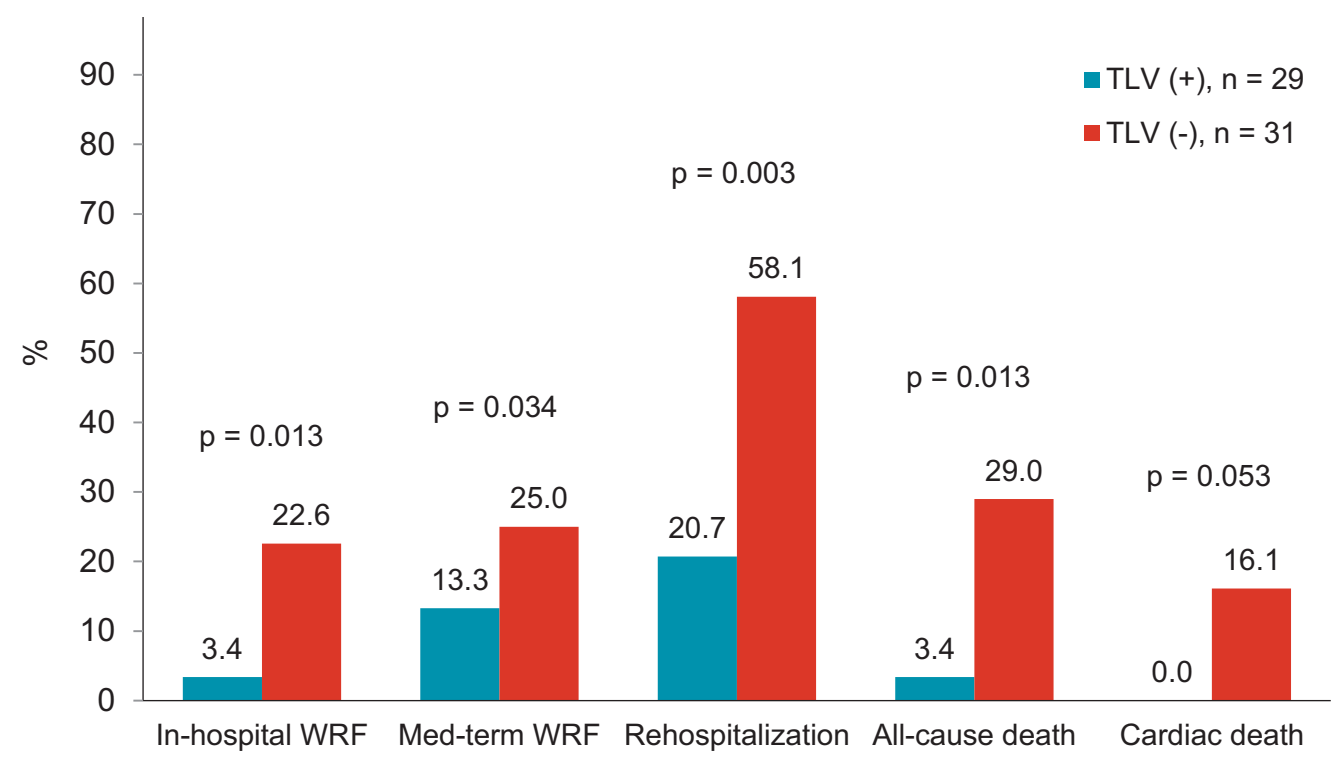

FIGURE 1. The incidence of WRF development, rehospitalization, all-cause death, and cardiac death in the study population. WRF - worsening renal function

\section{DISCUSSION}

This study demonstrated the efficacy of TLV therapy for elderly HFpEF patients. The main findings of the study were as follows: (1) in-hospital and mid-term WRF development was significantly lower in patients treated with TLV than in those not treated with TLV; (2) the bed rest

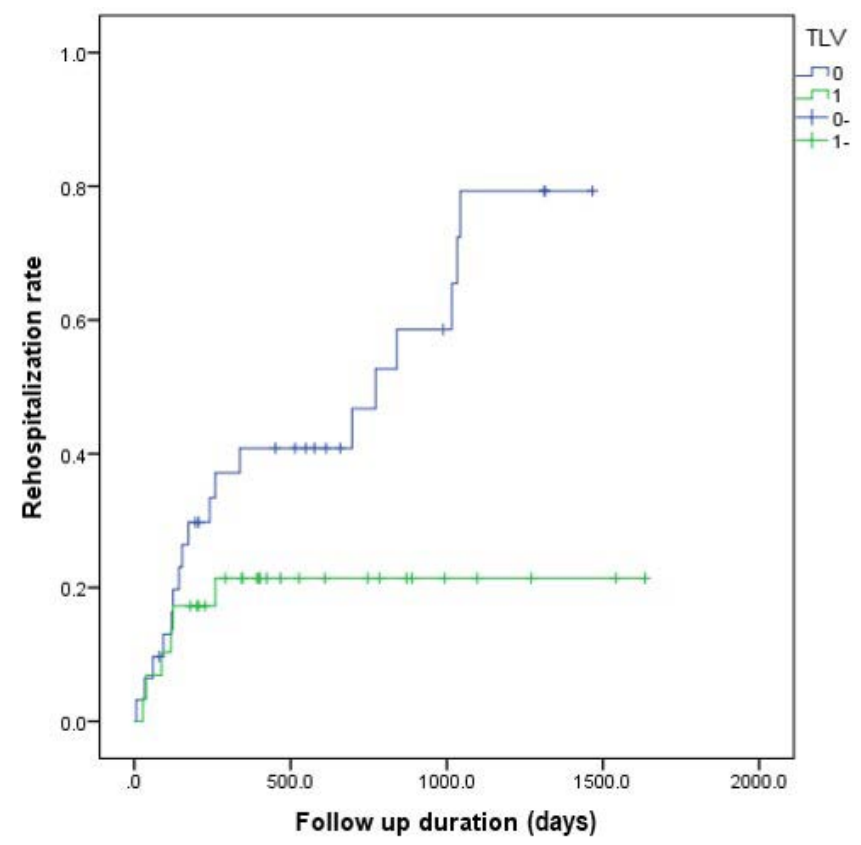

FIGURE 2. Cumulative incidence of rehospitalization in the study population. The incidence of rehospitalization was significantly lower in the TLV (+) group than in the TLV (-) group (log-rank test; $p=0.018) . T L V-$ tolvaptan period and the length of hospital stay were significantly shorter in patients treated with TLV therapy than in those not treated with TLV therapy; (3) the incidence of rehospitalization was significantly lower in patients treated with TLV therapy than in those not treated with TLV therapy; and (4) TLV therapy prevented the incidence of rehospitalization in patients with HFpEF.

\section{WRF DEVELOPMENT}

Previous studies have reported that WRF often occurred in subjects that had been admitted to the hospital for $\mathrm{HF}$ and was associated with an increased rate of adverse events. ${ }^{13,14}$ Preventive strategies towards decreasing WRF are important concerns, despite the fact that the underlying mechanisms of WRF are incompletely understood. Previous studies have shown that TLV improves systemic congestion without deteriorating the renal function. ${ }^{8,9,15-19}$ Inomata et al. reported that additive TLV was one of the independent predictors for preventing WRF during the course of treatment. ${ }^{15}$ Kimura et al. reported that early administration of TLV during hospitalization in elderly ADHF patients reduces WRF development, especially persistent and late-onset WRF development. ${ }^{8}$ Other studies have reported that TLV prevented WRF even in patients with HF and chronic kidney disease. ${ }^{18,19}$ In our study, $82.8 \%$ of the patients started TLV therapy from the first day of hospitalization, and TLV therapy reduced loop diuretics doses both at discharge and after 6 months, and it also prevented WRF development until 6 months 
TABLE 2. Multivariate logistic regression analysis

\begin{tabular}{|c|c|c|c|c|c|c|}
\hline & \multicolumn{3}{|c|}{ Univariate } & \multicolumn{3}{|c|}{ Multivariate } \\
\hline & OR & $95 \% \mathrm{CI}$ & $\mathrm{p}$ value & OR & $95 \%$ CI & $p$ value \\
\hline Female & 2.849 & $2.481-11.250$ & $<0.001$ & 2.861 & $2.032-8.382$ & 0.007 \\
\hline $\mathrm{AF}$ & 1.545 & $0.832-2.872$ & 0.193 & & & \\
\hline $\mathrm{DOA} / \mathrm{DOB}$ & 2.067 & $1.704-7.752$ & 0.021 & 2.559 & $1.176-6.723$ & 0.049 \\
\hline Carperitide & 1.091 & $0.528-2.253$ & 0.527 & & & \\
\hline ACE-I, ARB & 0.294 & $0.049-1.753$ & 0.206 & & & \\
\hline$\beta$-blocker & 0.994 & $0.525-7.041$ & 0.368 & & & \\
\hline $\mathrm{CCB}$ & 0.333 & $0.108-1.034$ & 0.064 & & & \\
\hline MRA & 0.657 & $0.269-1.602$ & 0.249 & & & \\
\hline TLV & 0.186 & $0.06-0.593$ & 0.004 & 0.191 & $0.116-0.717$ & 0.023 \\
\hline In-hospital WRF & 2.895 & $1.621-9.484$ & 0.047 & 1.97 & $0.118-7.815$ & 0.637 \\
\hline Med-term WRF & 2.806 & $1.296-6.078$ & 0.035 & 2.633 & $0.257-7.020$ & 0.415 \\
\hline
\end{tabular}

after discharge. Taken together, early and continuous administration of TLV seems to be an effective therapy for preventing WRF development and improving the clinical outcome in patients with HFpEF. However, in the TLV (+) group, the incidence of WRF development has increased from $3.4 \%$ at baseline to $13.3 \%$ at the 6 -month follow-up. The fact that the dose of loop diuretic increased after 6 months compared to the dose at discharge might indicate that TLV therapy could contribute to WRF development, and further studies are necessary to address this finding.

\section{BED REST PERIOD}

Older patients with HF are more likely to be hospitalized and are at risk of functional decline during hospitalization. A previous study has reported that there was a considerable loss of skeletal muscle, particularly from the lower extremities, as a result of bed rest. ${ }^{20}$ Uemura et al. demonstrated that physical activity at discharge independently predicted subsequent poor prognosis in HF patients. ${ }^{21}$ The prevention of functional decline during hospitalization is a very important issue, and the bed rest period should be as short as possible. Ueda et al. reported that an early switch from continuous intravenous infusion to oral medication significantly prevented the functional decline during hospitalization..$^{22}$ In our study, $82.8 \%$ of the patients started TLV therapy from the first day of hospitalization, and the duration of continuous intravenous infusion was significantly shorter in patients treated with TLV therapy. As a result, the bed rest period was significantly shorter in these elderly patients treated with TLV. These results suggest that TLV immediately improves systemic congestion and shortens both the bed rest period and the length of hospital stay, and might be able to prevent frailty in elderly patients with HF.

\section{REHOSPITALIZATION RATE}

Imamura and Kinugawa demonstrated that TLV therapy significantly decreased the incidence of rehospitalization in patients with HFpEF. ${ }^{23}$ Consistently, the current study demonstrated that TLV therapy reduced the incidence of rehospitalization in HFpEF patients, and TLV therapy after discharge was the predictive factor for rehospitalization. Previous studies have demonstrated the burden of recurrent congestion even after the initial relief of congestion during acute hospitalization. ${ }^{24,25}$ In both studies, clinical outcomes were superior for subjects with no or mild remaining congestion, while prognosis was significantly impaired in individuals with important remaining congestion at discharge. In this study, although there was no difference in the congestion score at discharge between the TLV (+) and TLV (-) groups, the congestion score was significantly higher in the TLV (-) group at 6 months after discharge. The ambulatory administration of TLV seems to continue to suppress recurrent congestion and prohibit the worsening of HF.

There are several limitations in this study. First, this was a retrospective observational study conducted at a single center, and the number of study participants was small; therefore, the statistical power might be low. Hence, the findings should be confirmed in a multi-center, large-scale, randomized study in the future. Second, each attending physician independently decided the timing and dosage of TLV, because an appropriate dose for each patient is essential for a successful HF management. 
Third, in administering TLV, it is often discussed whether the patients are "responders" or "non-responders." A previous study has reported that TLV might help to improve long-term prognosis in responders. ${ }^{23}$ In our study, patients treated with TLV showed clinical improvement and there seemed to be no non-responders.

\section{CONCLUSIONS}

In this study, the administration of TLV reduced the bed rest period, the length of hospital stay, and the incidence of rehospitalization without WRF development in elderly patients with HFpEF. TLV could be a safe and effective agent for the long-term management of this patient cohort.

\section{CONFLICT OF INTEREST}

Nothing to declare.

\section{REFERENCES}

1. Tsuchihashi-Makaya M, Hamaguchi S, Kinugawa S, et al. Characteristics and outcomes of hospitalized patients with heart failure and reduced vs preserved ejection fraction. Report from the Japanese Cardiac Registry of Heart Failure in Cardiology (JCARE-CARD). Circ J. 2009;73:1893-1900.

2. Hamaguchi S, Tsuchihashi-Makaya M, Kinugawa S, et al. Anemia is an independent predictor of long-term adverse outcomes in patients hospitalized with heart failure in Japan. A report from the Japanese Cardiac Registry of Heart Failure in Cardiology (JCARE-CARD). Circ J. 2009;73:1901-1908.

3. Suzuki S, Yoshihisa A, Yamaki T, et al. Acute heart failure volume control multicenter randomized (AVCMA) trial: comparison of tolvaptan and carperitide. J Clin Pharmacol. 2013;53:1277-1285. doi: 10.1002/jcph.197.

4. Ambrosy AP, Pang PS, Khan S, et al. Clinical course and predictive value of congestion during hospitalization in patients admitted for worsening signs and symptoms of heart failure with reduced ejection fraction: findings from the EVEREST trial. Eur Heart J. 2013;34:835-843. doi: 10.1093/ eurheartj/ehs444.

5. Kinugawa K, Sato N, Inomata T, Shimakawa T, Iwatake N, Mizuguchi K. Efficacy and safety of tolvaptan in heart failure patients with volume overload. Circ J. 2014;78:844-852.

6. Suzuki S, Yoshihisa A, Yamaki T, et al. Long-term effects and prognosis in acute heart failure treated with tolvaptan: the AVCMA trial. Biomed Res Int. 2014;2014:704289. doi: 10.1155/2014/704289.

7. Kinugawa $\mathrm{K}$, Inomata $\mathrm{T}$, Sato $\mathrm{N}$, et al. Effectiveness and adverse events of tolvaptan in octogenarians with heart failure. Interim analyses of Samsca Post-Marketing Surveillance In Heart faiLurE (SMILE study). Int Heart J. 2015;56:137-143. doi: 10.1536/ihj.14-332.

8. Kimura K, Momose T, Hasegawa T, et al. Early administration of tolvaptan preserves renal function in elderly patients with acute decompensated heart failure. J Cardiol. 2016;67:399405. doi: 10.1016/j.jjcc.2015.09.020.

9. Ono $\mathrm{Y}$, Takamatsu $\mathrm{H}$, Inoue $\mathrm{M}$, et al. Clinical effect of longterm administration of tolvaptan in patients with heart failure and chronic kidney disease. Drug Discov Ther. 2018;12:154160. doi: 10.5582/ddt.2018.01007.

10. Felker GM, Mentz RJ, Cole RT, et al. Efficacy and safety of tolvaptan in patients hospitalized with acute heart failure. J Am Coll Cardiol. 2017;69:1399-1406. doi: 10.1016/j. jacc.2016.09.004.

11. McKee PA, Castelli WP, McNamara PM, Kannel WB. The natural history of congestive heart failure: the Framingham study. N Engl J Med. 1971;285:1441-1446. doi: 10.1056/ NEJM197112232852601.

12. Ponikowski P, Voors AA, Anker SD, et al. 2016 ESC Guidelines for the diagnosis and treatment of acute and chronic heart failure. Kardiol Pol. 2016;74:1037-1147. doi: 10.5603/ KP.2016.0141.

13. Forman DE, Butler J, Wang Y, et al. Incidence, predictors at admission, and impact of worsening renal function among patients hospitalized with heart failure. J Am Coll Cardiol. 2004;43:61-67.

14. Krumholz HM, Chen YT, Vaccarino V, et al. Correlates and impact on outcomes of worsening renal function in patients $>$ or $=65$ years of age with heart failure. Am J Cardiol. 2000;85:1110-1113.

15. Inomata $\mathrm{T}$, Ikeda $\mathrm{Y}$, Kida $\mathrm{K}$, et al. Effects of additive tolvaptan vs. increased furosemide on heart failure with diuretic resistance and renal impairment - results from the K-STAR study. Circ J. 2017;82:159-167. doi: 10.1253/circj.CJ-17-0179.

16. Matsue Y, Suzuki M, Torii S, et al. Prognostic impact of early treatment with tolvaptan in patients with acute heart failure and renal dysfunction. Int J Cardiol. 2016;221:188-193. doi: 10.1016/j.ijcard.2016.07.063.

17. Matsue Y, Ter Maaten JM, Suzuki M, et al. Early treatment with tolvaptan improves diuretic response in acute heart failure with renal dysfunction. Clin Res Cardiol. 2017;106:802812. doi: 10.1007/s00392-017-1122-1.

18. Uemura Y, Shibata R, Takemoto K, et al. Safety and efficacy of long-term use of tolvaptan in patients with heart failure and chronic kidney disease. Circ J. 2017;81:1736-1738. doi: 10.1253/ circj.CJ-17-055.

19. Nakano $\mathrm{Y}$, Mizuno T, Niwa T, et al. Impact of continuous administration of tolvaptan on preventing medium-term worsening renal function and long-term adverse events in heart failure patients with chronic kidney disease. Int Heart J. 2018;59:105-111. doi: 10.1536/ihj.16-625.

20. Kortebein P, Ferrando A, Lombeida J, Wolfe R, Evans WJ. Effect of 10 days of bed rest on skeletal muscle in healthy older adults. JAMA. 2007;297:1772-1774. doi: 10.1001/jama.297.16.1772-b.

21. Uemura Y, Shibata R, Takemoto K, et al. Prognostic impact of the preservation of activities of daily living on postdischarge outcomes in patients with acute heart failure. Circ J. 2018;82:2793-2799. doi: 10.1253/circj.CJ-18-0279.

22. Ueda K, Kasao M, Shimamura $M$, et al. Impact of oral treatment on physical function in older patients hospitalized for heart failure: a randomized clinical trial. PLoS One. 2016;11:e0167933. doi: 10.1371/journal.pone.0167933.

23. Imamura $\mathrm{T}$, Kinugawa $\mathrm{K}$. Tolvaptan improves the long-term prognosis in patients with congestive heart failure with 
preserved ejection fraction as well as in those with reduced ejection fraction. Int Heart J. 2016;57:600-606. doi: 10.1536/ ihj.16-023.

24. Lucas C, Johnson W, Hamilton MA, et al. Freedom from congestion predicts good survival despite previous class IV symptoms of heart failure. Am Heart J. 2000;140:840-847. doi: 10.1067/mhj.2000.110933.
25. Lala A, McNulty SE, Mentz RJ, et al. Relief and recurrence of congestion during and after hospitalization for acute heart failure: insights from Diuretic Optimization Strategy Evaluation in Acute Decompensated Heart Failure (DOSEAHF) and Cardiorenal Rescue Study in Acute Decompensated Heart Failure (CARESS-HF). Circ Heart Fail. 2015;8:741-748. doi: 10.1161/CIRCHEARTFAILURE.114.001957. 